\title{
La literatura y el cine en la formación del médico y las humanidades médicas
}

\section{Literature and cinema in the training of doctors and medical humanities}

\author{
Orlando Mejia-Rivera. Manizales (Colombia)
}

DOI: https://doi.org/10.36104/amc.2019.1274

\begin{abstract}
Resumen
La utilización de la literatura y el cine para la enseñanza de valores humanistas a los estudiantes de medicina crea elementos conceptuales de gran importancia en el marco académico de las humanidades médicas. Las narrativas literarias y cinematográficas permiten generar en los alumnos una reflexión ética, histórica y epistemológica ante las perspectivas de la practica medica y la relación medico-paciente. Además, estimula en ellos las manifestaciones de solidaridad, empatía y reconocimiento del sufrimiento humano de los enfermos. En este artículo se muestran distintas orientaciones temáticas y obras especificas que pueden servir a la creación y desarrollo de cursos de pregrado en los currículos de medicina. (Acta Med Colomb 2019; 44. DOI: https://doi. org/10.36104/amc.2019.1274).
\end{abstract}

Palabras clave: literatura, cine, medicina, reflexión ética, alteridad, pedagogía.
Dr. Orlando Mejía Rivera: Profesor Titular del Programa de Medicina de la Facultad de Ciencias para la Salud de la Universidad de Caldas. Departamento de Salud Pública. Área de Humanidades Médicas. Médico. Especialista en Medicina Interna. Especialista en Literatura Hispanoamericana. Magíster en Filosofía con Énfasis en Epistemología. Escritor. Manizales (Colombia).

Correspondencia: Dr. Orlando Mejía-Rivera. Manizales (Colombia).

E-mail: paracelso2001@yahoo.com

Recibido: 8/X/2018 Aceptado: 22/VII/2019

\section{Introducción}

La enseñanza de la literatura como herramienta conceptual en la formación de los estudiantes de medicina de pregrado ya tiene una tradición significativa, que se remonta al curso pionero de la facultad de medicina de la Universidad de Pensilvania iniciado en el año de 1972 (1-3). A comienzos del siglo XXI 35\% de las facultades de medicina norteamericanas y europeas poseían ya un curso regular de esta temática en el currículo de sus programas (4-12) y desde el año 1982 existe la revista Literature and Medicine publicada por la Johns Hopkins University Press. Mi experiencia docente en esta área lleva más de diez años con el curso que dicto con el nombre de "La medicina y sus relaciones con la literatura, el arte y la filología" y que hace parte del área curricular de las Humanidades Médicas de mi escuela, en la cual soy el responsable [y que consta de cinco créditos obligatorios dentro del currículo y que está dividido así: historia de la medicina (dos créditos), dilemas éticos en la medicina contemporánea (dos créditos) y un crédito para el curso mencionado]. A continuación mostraré los fundamentos teóricos y las temáticas que se han desarrollado.

\section{La narrativa como instrumento de reflexión médica}

Con la ficción narrativa (cuentos y novelas) se construyen personajes y situaciones humanas que recrean la realidad, pero la expanden en su dimensión simbólica y en la variedad de sus significados. De ahí lo inolvidables que se tornan para los lectores ciertos personajes, que logran penetrar en la intimidad de las personas y se quedan allí para siempre. Esta capacidad de generar conocimiento y emoción proviene del poder de la narrativa para construir la alteridad (reconocimiento del otro) y establecer auténticos 
diálogos. Desde Bajtin sabemos que la novela moderna es "La percepción dialógica del mundo" (13) y ello engendra la "polifonía" narrativa, que no es más que la visión múltiple de la vida humana y la cual se contrapone a los discursos unilaterales que alimentan las ideologías totalitarias o los poderes únicos. En el contexto médico la narrativa permite el reconocimiento y comprensión de la perspectiva subjetiva del enfermo y de la familia ante el dolor y también enseña las implicaciones sociales (Sickness), personales (Ilness) y biomédicas (Disease) de la enfermedad. La existencia de estas tres dimensiones antropológicas de la enfermedad permiten al estudiante entender que la explicación biomédica de la patología del enfermo no le basta a él para comprender su dolencia y que debemos aprender el lenguaje y los simbolismos de la enfermedad como fenómeno subjetivo y cultural, al igual que como presencia colectiva y social $(14,15)$. Obras clásicas que sirven para este reconocimiento simultáneo de la enfermedad humana son, entre otras, La montaña mágica (1924) de Thomas Mann, La Peste (1947) de Albert Camus, Pabellón de cáncer (1967) de Aleksandr Solzhenitsyn, y una novela contemporánea y latinoamericana como La enfermedad (2006) de Alberto Barrera Tyszka.

\section{La voz del paciente}

Existen narraciones donde la presencia protagónica del personaje enfermo es central y autónoma. Esta experiencia brinda una gran lección de solidaridad a los estudiantes y les permite reconocer la existencia del enfermo que sufre y esto facilitará su actitud de respeto y empatía en las relaciones de su práctica clínica real. Como dice con acierto Luis Montiel, profesor de Humanidades Médicas de la Universidad Complutense de Madrid, en "la gran literatura el enfermo habla de sus miedos, de su dolor, de su impotencia, de su abandono, de su rencor, de su esperanza. Cualquiera que tenga alguna experiencia en el dolor humano sabe que lo que ese enfermo dice es verdadero, universalmente verdadero, aunque a la vez personal, íntimo, propio de quien de cierto modo ha vivido ante nosotros su existencia de ficción en el relato" (16). Entre las obras que mejor enfatizan en esta voz del enfermo sufriente y son convincentes, en mi experiencia docente, están: La muerte de Iván Ilich (1886) de León Tolstoi, Alguien voló sobre el nido del cuco (1962) de Ken Kesey, La campana de cristal (1963) de Sylvia Plath, Una muerte muy dulce (1964) de Simone de Beauvoir, Al amigo que no me salvó la vida (1991) de Hervé Guibert, La escafandra y la mariposa (2009) de Jean-Dominique Bauby.

\section{Médicos de ficción y autobiografías de médicos reales}

Los médicos de la ficción facilitan en los estudiantes la empatía con la profesión y son una fuente de emulación positiva, pues la mayoría de ellos son figuras heroicas que pertenecen al romanticismo y reflejan ambientes rurales o de medicina familiar que inspiran cercanías afectivas con los lectores jóvenes y en formación. Entre los clásicos están el Dr. Andrés Hurtado del Árbol de la ciencia (1911) de Pío Baroja, Esteban del Médico Rural (1912) de Felipe Trigo, el Dr. Andrew Manson de La Ciudadela (1939) de A.J. Cronin, el Dr. Carlo Levi (verdadero y desdoblado en personaje) de su novela autobiográfica Cristo se detuvo en Éboli (1946), el Dr. Michel Doutreval de Cuerpos y Almas (1943) de Maxence Van Der Meersch, Pedro de Tiempo de silencio (1962) de Luis Martín-Santos, Juvenal Urbino de El amor en los tiempos del cólera (1985) de García Márquez, Roy Bash de La casa de Dios (2002) de Samuel Shem, los hermanos Stone de Hijos del ancho mundo (2009) de Abraham Verghase. También están las novelas históricas con médicos inventados o recreados como Sinuhé de Sinuhé el egipcio (1945) de Mika Waltari, el evangelista Lucas de Médico de cuerpos y almas (1958) de Taylor Caldwell, el Maimónides de El Médico de Córdoba (1977) de Herbert Le Porrier, Rob Cole de El Médico (1986) de Noah Gordon, el Avicena de La ruta de Isfahan de Gilbert Sinoué. Estos médicos históricos ficcionalizados ayudan a conocer la historia de la medicina de diversas épocas y la lectura de estas obras se pueden complementar con textos históricos o biográficos del mismo periodo analizado. Por ejemplo, la novela de Sinoué se puede leer de forma simultánea con los ensayos de Soheil Afnan y El pensamiento de Avicena (1965), Miguel Cruz Hernández y La metafísica de Avicena (1949) o Rafael Ramón Guerrero y Avicena (1996).

Las autobiografías de médicos reales generan una gran discusión y reflexión entre los estudiantes y algunas de ellas son preferidas, incluso, a otros textos de ficción. Quizá porque se convierten en modelos auténticos que buscan ser imitados por los alumnos. El género de la autobiografía científica tiene su origen en la obra de David Hume titulada De mi propia vida (1776) y se caracteriza por entremezclar la vida personal y los logros científicos, en lo que se conoce como contexto del descubrimiento y que ha sido estimulado por filósofos de la ciencia como Thomas Kuhn y su famoso libro La estructura de las revoluciones científicas (1961) (17). Las autobiografías médicas incorporan la experiencia clínica que tuvieron sus autores con los enfermos y este aspecto enriquece la visión que adquieren los estudiantes de la relación médico-paciente y también de cómo algunos descubrimientos clínicos surgieron de reflexionar sobre los casos clínicos que les tocó tratar. Autobiografías clásicas como La historia de San Michelle (1939) de Axel Munthe o la trilogía del premio Nobel de medicina Santiago Ramón y Cajal: Infancia y juventud (1901), Historia de mi labor científica (1923) y El mundo visto a los ochenta años (1934) se combinan bien con obras autobiográficas contemporáneas como La estatua interior (1987) del genetista francés y premio Nobel Francois Jacob, El Tío Tungsteno: Recuerdos de un Químico Precoz (2003) y la conmovedora En movimiento. una vida (2015), ambas del neurólogo Oliver Sacks o la reciente Ante todo no hagas daño (2016) del neurocirujano inglés Henry Marsch. Palabras como las siguientes de Sacks, de su ensayo de publicación póstuma 
titulado Gratitud (2016), son una lección de integridad y valentía ante la propia enfermedad que enseña para toda la vida a los estudiantes: "A los ochenta y un años, seguía nadando kilómetro y medio cada día. Pero se me ha acabado la suerte: hace una semana me comunicaron que padezco metástasis múltiples en el hígado. Hace nueve años me descubrieron en el ojo un tumor poco común, un melanoma ocular. El tratamiento con radiación y láser para eliminar el tumor acabó por dejarme ciego de ese ojo. (...) Doy gracias porque se me hayan concedido nueve años de buena salud y productividad desde el diagnóstico original, pero ahora veo la muerte cara a cara. Ahora me toca decidir cómo quiero vivir los meses que me quedan. Tengo que vivirlos de la manera más rica, intensa y productiva que pueda..." (18). Y concluye con estas valientes y profundas reflexiones: "Y ahora, débil, sin aliento, con los músculos antaño firmes reblandecidos por el cáncer, descubro que mis pensamientos cada vez giran menos en torno a lo sobrenatural o espiritual y más en torno a lo que significa llevar una vida buena y que merezca la pena, alcanzar una sensación de paz con uno mismo. Me descubro pensando en el sabbat, el día de descanso, el séptimo día de la semana, y quizá también el séptimo día de la propia vida, cuando tienes la sensación de que tu obra está terminada y de que, con la conciencia tranquila, puedes descansar" (19).

\section{Anton Chéjov y los cuentos de médicos}

El mejor ejemplo de un médico escritor es Anton Chéjov (1860-1904), quien ejerció la medicina durante la mayor parte de su corta vida y de manera simultánea escribió una obra literaria inmortal, hasta el punto de ser considerado el precursor del cuento moderno en Occidente, al lado de Edgar Allan Poe. Además, su condición personal de tuberculoso crónico le agrega un componente dramático a varios de sus personajes enfermos. Se puede seleccionar una serie de cuentos y dramas de temas médicos de Chéjov, para hacer un curso exclusivo con la literatura del ruso. Por ejemplo, esta lista tendría como relato central el extraordinario cuento titulado El pabellón número 6, que describe la relación entre el doctor Andréi Yefímych Raguin y el enfermo mental, con manía persecutoria, de nombre Iván Dmítrich Grómov. Aquí es tratado con maestría inigualable el tema de la empatía en la relación médico-paciente y el reconocimiento del sufrimiento de los pacientes. Este texto sigue siendo impactante para los estudiantes y les genera múltiples sensaciones y reflexiones. Otras obras con médicos y enfermedades en Chéjov que complementan el curso son: el Dr. Triletski del drama Platanov, el Dr Lvov del drama Ivanov, el Dr Astrov del drama Tío Vania, el Dr. Dorn del drama La Gaviota. Cuentos como Perpetum Mobile, Los nervios, Una crisis nerviosa, Enemigos, Tifo, El monje negro, La esposa, Tres años, etcétera. En la totalidad de la obra de Chéjov existen treinta y seis personajes médicos y los textos de temática médica alcanzan la cifra de cuarenta y cinco $(20,21)$. No en vano Chéjov escribió en el año de 1900 lo siguiente:
"No dudo que mis actividades como médico han tenido una fuerte influencia en mi trabajo como escritor. Han ampliado significativamente mi campo de observación, enriquecido mi conocimiento, y sólo las personas que son médicos pueden apreciar el valor que todo esto tiene. La medicina ha sido también una guía, y probablemente no he llegado a cometer muchos errores como resultado de mi estrecha relación con ella" (22). Otros cursos dedicados a un solo autor pueden ser la lectura de las novelas y cuentos sobre el doctor de ficción Sherlock Holmes del médico escocés Arthur Conan Doyle, que además permiten una reflexión paralela entre el arte del diagnóstico clínico y la investigación detectivesca $(23,24)$. Además, los cuentos del libro Historias de médicos (1995) del poeta y clínico norteamericano William Carlos Williams pueden ser un interesante curso temático, que se podría complementar con fuentes históricas del ejercicio de la medicina rural durante la primera mitad del siglo XX como, por ejemplo, el sugestivo libro de memorias del médico colombiano Jaime Mejía Mejía titulado Historias médicas de una vida y una región (1960).

\section{Oliver Sacks y la invención de un subgénero literario-clínico}

Oliver Sacks, médico neurólogo nacido en 1933 y fallecido en 2015, ha sido considerado el mejor escritor clínico de la época actual. Su obra es una afortunada fusión de la experiencia como médico práctico y su capacidad para narrar esos extraños mundos de los pacientes, logrando así la humanización de los enfermos, pues al describir sus historia personales de vida inmersas en sus distintas patologías, los lectores comprendemos que la enfermedad es, casi siempre, el puente obligado para pasar a otra realidad donde cada enfermo debe reacomodar su existencia de acuerdo con sus nuevas limitaciones corporales. En el prólogo a su famoso libro El hombre que confundió a su mujer con un sombrero, Sacks sintetizó su propuesta clínico-literaria así: "En un historial clínico riguroso no hay "sujeto"; los historiales clínicos modernos aluden al sujeto con una frase rápida ("hembra albina trisómica de 21”), que podría aplicarse igual a una rata que a un ser humano. Para situar de nuevo en el centro al sujeto (el ser humano que se aflige y que lucha y padece) hemos de profundizar en un historial clínico hasta hacerlo narración o cuento; sólo así tendremos un "quién" además de un "qué", un individuo real, un paciente, en relación con la enfermedad...en relación con el reconocimiento médico físico" (25).

Es decir, la obra de Sacks es la prueba de que el rigor científico se hace comprensible y transmisible con el pleno reconocimiento de la rica individualidad de cada enfermo. Conocer, entonces, las historias clínicas descritas por el doctor Sacks es una vía maravillosa para que los estudiantes de medicina entiendan que la clínica es el arte de diagnosticar y tratar a personas complejas, únicas, y cuyos síntomas y signos serán mejor entendidos si sabemos de su vida personal. A esta nueva propuesta la denominó Sacks "Clinical 
Tales" (26) y aunque en sentido estricto la traducción más literal al español sería "cuentos clínicos" pienso que para diferenciarlos de, por ejemplo, los cuentos de Chéjov podrían denominarse "relatos clínicos" y esta titulación abarca de mejor manera el componente real de las historias descritas, pero con las estructuras formales de la narrativa.

El curso selecciona varios relatos de sus distintos libros y los estudiantes divididos en grupos exponen cada relato y, a la vez, exploran la parte clínica y científica de los mismos, recurriendo a la medicina interna de Harrison o a la neurología de Adams. Un ejemplo de la serie de relatos escogidos es la siguiente: El hombre que confundió a su mujer con un sombrero, Vida de un cirujano, El caso del pintor ciego al color, El marinero perdido, Las visiones de Hildegard, Alucinaciones musicales, Dedos fantasmas: El caso del pianista con un solo brazo, A nivel, Manos y prodigios, Un mundo auditivo: música y ceguera, Una especie hipermusical: el síndrome de Williams, El paisaje de sus sueños (se les hace leer también el cuento de Jorge Luis Borges titulado Funes el memorioso), El último hippie, Ver y no ver. Los libros de donde se han extraído éstas y otras historias son: Los ojos de la mente (2011), Musicofilia (2009), El tío Tungsteno (2001), El hombre que confundió a su mujer con un sombrero (2000), La isla de los ciegos al color (1997), Un antropólogo en Marte (2004), Veo una voz: viaje al mundo de los sordos (2003), Con una sola pierna (2003), Despertares (1974), Migraña (1997).

El subgénero de los "relatos clínicos" es una fuente de riqueza humanista y médica, que ha sido muy bien recibida por los alumnos y también ha motivado a otros médicos a expresar por escrito sus experiencias clínicas mediante las formas narrativas. Aquí resalto otro libro titulado El hombre con el tatuaje de hierro. Los médicos aprenden de sus pacientes (2009). Cuyos autores son los neurólogos norteamericanos John Castaldo y Lawrence P. Levitt y quienes en el prólogo de su obra refieren que: "A medida que íbamos aprendiendo más sobre estas alteraciones neurológicas y cómo tratarlas, empezamos a darnos cuenta de que nuestros pacientes también nos proporcionaban más sabiduría -sabiduría sobre cómo vivir-. También aprendíamos mucho de los familiares que, a menudo, se enfrentaban a cambios desgarradores en los seres queridos y, por tanto, en su rol y sus ritmos cotidianos" (27).

\section{Otras temáticas}

El curso de literatura y medicina también se puede orientar al enfoque de otras relaciones como: las descripciones clínicas de enfermedades en las obras literarias (La enfermedad de Sach (1999) de Martín Winckler, Los papeles póstumos del club Pickwick (1837) de Charles Dickens, Doktor Faustus (1947) de Thomas Mann, El enfermo de Abisinia (2007) de Orlando Mejía Rivera, etcétera), la interpretación semiológica de los síntomas y signos descritos por los grandes maestros de la narrativa universal, la percepción de las etapas vitales de los seres humanos, la descripción de epidemias y pandemias (El Decamerón (1350) de Giovanni Bocaccio, El diario del año de la peste (1722) de Daniel Defoe, Ensayo sobre la ceguera (1995) de José Saramago, El libro del día del juicio final (1992) de Connie Willis, La amenaza de Andrómeda (1969) de Michael Crichton, Némesis (2010) de Philip Roth, Peste \& Cólera (2012) de Patrick Deville, etcétera).

\section{Cine y medicina}

El cine es otra herramienta conceptual interesante y apasiona a los estudiantes. Los mismos objetivos y temáticas de la literatura sirven para la enseñanza de problemáticas médicas a través del cine (28-32). Se pueden ofrecer varios ciclos temáticos y el espacio ideal de presentación es el de la creación de un cine-foro semanal o quincenal o mensual (auspiciado por la facultad y la oficina de educación médica) y las películas se dan en un ámbito transcurricular, que motiva los diversos diálogos entre estudiantes de distintos semestres y los mismos docentes de los diferentes departamentos de la facultad. Se recomiendan fuentes bibliográficas como La revista de cine y medicina (2005-2016) de la Universidad de Salamanca (con varios artículos de pedagogía del cine en medicina y recomendaciones de películas de ámbito médico) y libros teóricos como Med\&Cine: Encuentros, tribulaciones y cortocircuitos entre cine y medicina (1998) de Jordi Batlle Caminal; Médicos en el cine: dilemas bioéticos: sentimientos, razones y deberes (2006) de Diego Gracia y Sagrario Muñoz Calvo; Cine y medicina (2008) de Fernando Giménez Escribano; Bioética y cine. De la narración a la deliberación (2011) de Tomás Domingo Moratalla; Mayores de Cine. El cine como instrumento de aprendizaje en gerontología (2012) de Juan Dionisio Hernández Avilés y Terapias de cine. 50 películas básicas en torno a la medicina (2016) de Carlos Tabernero Holgado.

De algunas obras en inglés que también fundamentan distintos aspectos de la relación cine-medicina se recomiendan: Psychiatry and the Cinema (1999) de Glen O. Gabbard y Krin Gabbard; Movies And Mental Illness: Using Films To Understand Psychopathology (2005) de Danny Wedding y Mary Ann Boyd; Mediciness Moving Pictures: Medicine, Health, and Bodies in American Film and Television (2008) de Leslie J. Reagan y Nancy Tomes; Legacies of Plague in Literature, Theory and Film (2009) de J. Cooke; The Picture of Health: Medical Ethics and the Movies (2011) de Henri Colt y Silvia Quadrelli; The Dark Mirror: Psychiatry and Film Noir (2011) de Marlisa Santos; Psychoanalysis and Film (2011) de Glen O. Gabbard; Medical Visions: Producing the Patient Through Film, Television, and Imaging Technologies (2013) de Kirsten Ostherr; Neurocinema: When Film Meets Neurology (2014) de Eelco F. M. Wijdicks; Movies and Mental Illness: Using Films to Understand Psychopathology (2014) de Danny Wedding and Ryan M. Niemiec; Neuroscience in Science Fiction Films (2014) de Sharon Packer y Monsters, Demons and Psychopaths: Psychiatry and Horror (2016) de Fernando Espi Forcen. 
Por último las sugerencias para iniciar un curso de cine y literatura, mediante el formato de cine-foro, con una película quincenal y de temas variados serían las siguientes: Despertares/ Awakenings (1990) de Penny Marshall (basado en el libro del mismo nombre de Oliver Sacks y que trata de las historias de un grupo de enfermos crónicos con secuelas neurológicas de la epidemia de encefalitis letárgica de 19171928 y sus mejorías transitorias al recibir la droga L-Dopa); El experimento Tuskgee/ Miss Evers 'Boys (1977) de Joseph Sargent (recrea la terrible transgresión ética realizada con pacientes afroamericanos con sífilis que nunca recibieron el tratamiento con penicilina entre los años de 1932 y 1975); Amar la vida/ Wit (2001) de Mike Nichols, Las invasiones bárbaras/ Les invasions barbares (2003) de Denys Arcand y Mar adentro (2004) de Alejandro Amenábar (tres clásicos contemporáneos notables del dilema de los enfermos en estado terminal, los cuidados paliativos y la eutanasia); Philadelphia (1993) de Jonathan Demme (el tema de la discriminación a los enfermos de SIDA al inicio de la epidemia); El hombre elefante/ The Elephant Man (1980) de David Lynch (la historia real de Joseph Merrick y su sufrimiento debido a la neurofibromatosis severa que padeció); Mi pie izquierdo/ My left foot (1989) de Jim Sheridan (sobre el caso de Christy Brown, una persona con parálisis cerebral); Una mente maravillosa/ A Beautiful Mind (2001) de Ron Howard (sobre la vida de John Forbes Nash, premio Nobel de economía a pesar de su diagnóstico de esquizofrenia); El hijo de la novia (2001) de Juan José Capanella, Lejos de ella/ Away from Her (2006) de Sarah Polley y Siempre Alice/ Still Alice (2014) de Wash Westmoreland y Richard Glatzer (tres buenas películas sobre enfermos con demencia de Alzheimer).

\section{Conclusiones}

La literatura y el cine son instrumentos poderosos y convincentes en la pedagogía de las humanidades médicas. Permiten una interrelación íntima con la reflexión bioética, la historia de la medicina y la epistemología médica y contribuyen a generar en los estudiantes de medicina una actitud solidaria con los pacientes, sus familias y los colegas. En síntesis, la narrativa literaria y cinematográficas son herramientas excelsas para lograr el reconocimiento de la alteridad. Como bien dijeron Ann Hunsaker Hawkins y Marilyn Chandler McEntyre, la enseñanza de la literatura a los alumnos de medicina "es una vía única para imaginar al otro, para usar la imaginación como instrumento de la compasión, para tolerar la ambigüedad, para convivir con lo paradójico, para considerar múltiples puntos de vista y para reconocer las distintas verdades que poseen las experiencias humanas" (33).

\section{Referencias}

1. Trautmann J. The wonders of literature in medical education. J of Contin Educ Health 1982; 2: 23-31.

2. Trautmann J. Can we resurrect Apollo?. Lite Med . 1982; 1: 1-18.
3. Charon R., Banks J. T., Connelly J. E., Hawkins A. H., Hunter K. M., Jones A.H. Literature and medicine: contributions to clinical practice. Ann Intern Med; 1995;122: 599-606.

4. Hawkins AH, McEntyre MC. Introduction: Teaching literature and medicine: a retrospective and a rationale. En Hawkins AH, McEntyre MC (eds.) Teaching literature and medicine. New York: The Modern Language Association. 2000. p $1-28$.

5. Skelton JR,Thomas CP, Macleod JAA. Teching literature and medicine to medical students, part I: the beginning. The Lancet 2000; 356:1920-1922.

6. Skelton JR, Thomas CP, Macleod JAA. Teaching literature and medicine to medical students, part II: why literature and medicine? The Lancet 2000; 356: 2001-2003.

7. Hurwitz B. Narrative and the practice of medicine. The Lancet. 2000; 356: 20862089.

8. Rabuzzi K, ed. Toward a new discipline. Lit Med. 1982;1: 1.

9. Downie RS. Literature and medicine. J Med Ethics. 1991;17: 93-96.

10.Caiman KC, Downie RS, Duthie M, Sweeney B. Literature and medicine: a short course for medical students. Med Educ. 1988; 22: 265-699.

11. Charon R, Banks JT, Connelly JE, Hawkins AH, Hunter KM, Jones AH, et al. Literature and medicine: contributions to clinical practice. Ann Intern Med. 1995; 122: 599-606.

12. McLellan MF, Jones AH. Why literature and medicine? The Lancet. 1996; 348:109-111.

13. Bajtin MM. Problemas de la poética de Dostoievski. Bogotá: FCE. 1993. p 374.

14. Young A. The anthropologies of illness and sickness. Ann. Rev. Anthropol. 1982; 11: 257-285.

15. Hernáez AM. Antropología Médica. Teorías sobre la cultura, el poder y la enfermedad. Barcelona: Anthropos. 2008. p 80-81.

16. Montiel L. Medicina y Literatura. (Conferencia Inaugural del XXI Congreso Nacional de SEMERGEN). SEMERGEN, 1999; XXV, nº extraordinario (XXI Congreso Nacional de la Medicina Rural y Generalista): 21-26.

17. Salvador V. Autorrelato e identidades profesionales. Sobre autobiografías de científicos y médicos. Annali di Ca' Foscari. 2015; 49: 57-74.

18. Sacks O. Gratitud. Barcelona: Anagrama. 2016. p 9-10.

19. Sacks O. Gratitud. Barcelona: Anagrama. 2016. p 60-61.

20. Crommelynck I. Doctor Chekhov's Doctors. En: J. Bogousslavsky, $\square$ S. Dieguez (eds) Literary Medicine: Brain Disease and Doctors in Novels, Theater, and Film. Front Neurol Neurosci. 2013; 31: 236-244.

21. Cohen B. Anton Chekhov (1860-1904)--a 19th century physician. J Med Biogr. 2007; 15:166-73

22. Bartlett R. Chéjov: Escenas de una vida. Madrid: Siglo XXI. p 84.

23. Ginzburg C. Morelli, Freud y Sherlock Holmes: Indicios y método científico. En: Eco U, Sebeok TA (Eds). El signo de los tres. Dupin, Holmes, Peirce. Barcelona: editorial Lumen. 1989. p 116-163.

24. Fitzgerald FT, Tierney Jr, LM. The bedside Sherlock Holmes. West J. Med. 1982; 137:169-175.

25.Sacks O. El hombre que confundió a su mujer con un sombrero. Barcelona: Muchnik editores. 2000. p 12.

26. Sacks O. Clinical Tales. Lit Med. 1986; 5:16-23.

27. Castaldo JE, Levitt LP. El hombre con el tatuaje de hierro. Los médicos aprenden de sus pacientes. Barcelona: Plataforma Editorial. p 20.

28. Astudillo W, Mendinueta C. El cine en la docencia de la medicina: cuidados paliativos y bioética. Rev Med Cine. 2007; 1:32-41

29. Martínez, MJF, Amado CD, Sánchez EG, Sánchez JEG. Metodología docente para la utilización del cine en la enseñanza de la microbiología médica y las enfermedades infecciosas. Rev Med Cine. 2005; 1:17-23.

30. Mercadé AB. Bioética clínica y narrativa cinematográfica. Rev Med Cine. 2005; 1:77-81.

31. Carrió ER. De Cameron a Haneke, un punto inicial para analizar el cine gerontológico. Rev Med Cine. 2015; 11:105-108.

32. Farre M, Arribas S, Pérez J, Baños JE. El uso de películas comerciales para comprender los principios bioéticos en la investigación clínica. Rev Med Cine. 2013; 9:151-155.

33. Hawkins AH, McEntyre MC. Introduction: Teaching literature and medicine: a retrospective and a rationale. En Hawkins AH, McEntyre MC (eds.) Teaching literature and medicine. New York: The Modern Language Association. 2000. p 14. 